\title{
Editorial
}

\section{Is interleukin-1 a good target for therapeutic intervention in intervertebral disc degeneration: lessons from the osteoarthritic experience}

\author{
Philippe Goupille ${ }^{1}$, Denis Mulleman ${ }^{1}$ and Xavier Chevalier ${ }^{2}$
}

\author{
${ }^{1} \mathrm{CHRU}$ de Tours, Université François Rabelais, INSERM, Centre d'investigation clinique 202, Tours, France \\ 2University Hospital, Créteil, France
}

Corresponding author: Philippe Goupille, goupille@med.univ-tours.fr

Published: 21 November 2007

Arthritis Research \& Therapy 2007, 9:110 (doi:10.1186/ar2324)

This article is online at http://arthritis-research.com/content/9/6/110

c) 2007 BioMed Central Ltd

See related research article by Le Maitre et al., http://arthritis-research.com/content/9/4/R77

\begin{abstract}
IL-1 plays a key role in disc degeneration and could be a valid target for inhibiting this process. IL-1 receptor antagonist (IL-1 ra) might be a good candidate to inhibit IL-1 activity. However, many questions need to be addressed before contemplating therapy in humans. IL-1 blockade is also a great challenge in osteoarthritis and results from animal models suggest that IL-1 ra may have beneficial effects. The clinical benefit of a local injection of IL-1 ra in knee osteoarthritis may be limited by the antagonist's short half-life. Further studies with longer-lasting antagonists are needed to explore this new therapeutic approach.
\end{abstract}

A better understanding of the pathogenesis of inflammatory arthritis has led to the development and subsequent successful use in humans of biopharmaceuticals blocking, for example, tumor necrosis factor (TNF) $\alpha$, CD-20, IL-1 and IL-6 and T-cell co-stimulating factors (CTLA-4). No targeted treatments are available, however, for degenerative rheumatological diseases such as low back pain (LBP) due to intervertebral disc (IVD) degeneration or knee osteoarthritis (OA). This deficiency may be due in part to our limited understanding of the pathogenesis of these diseases; a better understanding of the pathogenesis of IVD degeneration is a prerequisite for developing successful biological therapeutic approaches for patients with LBP.

Current work presented by Freemont's group [1] has contributed greatly to our understanding of disc remodeling in degenerative IVD, notably the role of pro-inflammatory cytokines. They compare the expression of IL-1 $\beta$ and TNF $\alpha$ as well as their main functional receptors in human IVDs [1]. The most important aspect of this study is the comparison of non-degenerated, degenerated and herniated samples of human IVDs. In non-degenerated human IVDs, both cytokines were expressed at low levels, with an increase in IL-1 $\beta$ compared to TNF $\alpha$ and a low level of cells positive for TNF receptor type I [1]. In degenerated human IVDs, although the protein production of the two cytokines was increased compared with the non-degenerated samples, the number of positive cells and the level of gene expression was greater for IL-1 $\beta$ than for TNF $\alpha$. Furthermore, IL-1 RI gene expression and protein production were also increased in degenerated compared with non-degenerated samples, and a tendency for decreased synthesis of TNF RI was even observed in herniated and degenerated human IVDs compared to nondegenerated samples. These results are very important because they suggest that IL- $1 \beta$ is more predominant in the process of IVD degeneration than TNF $\alpha$ [2]. However, they do not explain the exact roles of both cytokines and raise more questions than they answer. How do the effects of TNF $\alpha$ and IL-1 $\beta$ combine to alter the extracellular matrix of discs? Do they act in the same way in degenerated and herniated IVDs? Do they act in the same time period? Are both cytokines involved in pain generation? What is the role of IL-1 $1 \alpha$ ?

These data suggest that IL-1 $\beta$ could be a valid target for inhibiting disc degeneration and offer an exciting challenge for developing future therapeutic approaches. Among IL-1 inhibitors, IL-1 receptor antagonist (IL-1 ra) might be a candidate for preventing IVD degeneration [3]. Indeed, Freemont and colleagues have shown in monolayer and three-dimensional alginate-cultured resident cells from degenerate IVDs that IL-1 ra down-regulates metal-dependent proteases [4] and, delivered directly or by gene therapy in explants of degenerated human IVDs, almost completely eliminates enzyme activity, thereby decreasing extracellular matrix degradation [2].

$\mathrm{IL}=$ interleukin; IL-1 ra = IL-1 receptor antagonist; IVD = intervertebral disc; LBP = low back pain; OA = osteoarthritis; TNF = tumor necrosis factor. 


\section{What can we learn from the trials performed in osteoarthritis with IL-1 $\beta$ blockers?}

It is indeed tempting to compare IVD degeneration to OA because the profile of cytokine production in human IVDs looks like the one observed in OA and because resident cells from discs behave like chondrocyte cells. Some evidence suggests that $\mathrm{IL}-1 \beta$ is not only involved in the structural damage process of OA but also plays an important role in pain transmission. Results from in vitro studies and animal models of OA support the dominant role of IL-1 $\beta$ early in the disease process. Moreover, intra-articular delivery of anakinra (recombinant methionyl human receptor antagonist $(r-m e t$ HulL-1ra)) may have beneficial effects on symptoms and structural modifications in animal models of OA [5-7]. Treated animals in these studies showed less severe cartilage lesions, less synovitis, significant reduction in the size of osteophytes, and significant improvement in clinical indicators of pain and disease activity.

A first randomized controlled trial in patients with knee OA demonstrated a good safety profile for one intra-articular injection of IL-1 ra (150 mg, the maximum tolerated dose) [8]. We performed a multicenter, randomized, double-blind, placebo-controlled study to evaluate the clinical response, safety, and tolerability of a single intra-articular injection of anakinra (50 or $150 \mathrm{mg}$ ) in 170 patients with symptomatic OA of the knee [9]. There was no improvement in knee pain, function, or measures of cartilage turnover with anakinra treatment compared to placebo at the main endpoint (month 1). A tendency for improvement was noticed in the $150 \mathrm{mg}$ group at day 4 (in keeping with the short half-life of IL-1 ra), suggesting that IL-1 inhibition may be therapeutically relevant.

In the context of LBP, the use of IL-1 blockers is very attractive, although many questions need to be addressed before starting to use such therapy: what is the best route of administration, how many injections should be given, what types of IL-1 blockers should be used, and at what stage of the disease should therapy be given?

\section{Competing interests}

The authors declare that they have no competing interests.

\section{References}

1. Le Maitre CL, Hoyland JA, Freemont AJ: Catabolic cytokine expression in degenerate and herniated human intervertebral discs: IL-1 $\beta$ and TNF $\alpha$ expression profile. Arthritis Res Ther 2007, 9:R77.

2. Le Maitre CL, Hoyland JA, Freemont AJ: Interleukin-1 receptor antagonist delivered directly and by gene therapy inhibits matrix degradation in the intact degenerate human intervertebral disc: an in situ-zymographic and gene therapy study. Arthritis Res Ther 2007, 9:R83.

3. Le Maitre CL, Hoyland JA, Freemont AJ: The role of interleukin1 in the pathogenesis of human intervertebral disc degeneration. Arthritis Res Ther 2005, 7:R732-R745.

4. Le Maitre $\mathrm{CL}$, Hoyland JA, Freemont $\mathrm{AJ}$ : $\mathbf{A}$ preliminary in vitro study into the use of IL-1Ra gene therapy for the inhibition of intervertebral disc degeneration. Int J Exp Pathol 2006, 87:1728.
5. Caron JP, Fernandes JC, Martel-Pelletier J, Tardif G, Mineau F, Geng C, Pelletier JP: Chondroprotective effect of intra-articular injections of interleukin-1 receptor antagonist in experimental osteoarthritis. Suppression of collagenase-1 expression. Arthritis Rheum 1996, 39:1535-1544.

6. Pelletier JP, Caron JP, Evans C, Robbins PD, Georgescu HI, Jovanovic D, Fernandes JC, Martel-Pelletier J: In vivo suppression of early experimental osteoarthritis by interleukin-1 receptor antagonist using gene therapy. Arthritis Rheum 1997, 40:1012-1019.

7. Fernandes JC, Tardif G, Martel-Pelletier J, Lascau-Coman V, Dupuis M, Moldovan F, Sheppard M, Krishnan BR, Pelletier JP: In vivo transfer of interleukin-1 receptor antagonist gene in osteoarthritic rabbit knee joints: prevention of osteoarthritis progression. Am J Pathol 1999, 154:1159-1169.

8. Chevalier X, Giraudeau B, Conrozier T, Marlière J, Kiefer P, Goupille P: Safety study of intraarticular injection of interleukin 1 receptor antagonist: a multicenter study. I Rheumatol 2005, 32:1317-1323.

9. Chevalier X, Goupille P, Beaulieu AD, Burch FX, Conrozier T, Loeuille D, Kivitz AJ, Silver D, Kiefer P, Zhou L, et al.: Results from a double blind, placebo-controlled, multicenter trial of a single intra-articular injection of Anakinra $\left(\right.$ Kineret ${ }^{\mathbb{R}}$ ) in patients with osteoarthritis of the knee. Arthritis Rheum 2005, 52(Suppl 9):S507. 